\title{
Obtaining Full-Diversity Space-Frequency Codes From Space-Time Codes via Mapping
}

\author{
Weifeng Su, Member, IEEE, Zoltan Safar, Masoud Olfat, Student Member, IEEE, and K. J. Ray Liu, Fellow, IEEE
}

\begin{abstract}
This paper considers the problem of space-frequency code design for frequency-selective multiple-input-multiple-output (MIMO) orthogonal frequency division multiplexing (OFDM) modulation. We show that space-time codes achieving full diversity in quasistatic flat fading environment can be used to construct space-frequency codes that can achieve the maximum diversity available in frequency-selective MIMO fading channels. Since the codes are constructed via a simple mapping from space-time codes to space-frequency codes, the abundant classes of existing space-time block and trellis codes can be used for full diversity transmission in MIMO-OFDM systems. The proposed mapping provides a tradeoff between the achieved diversity order and the symbol rate. Moreover, we characterize the performance of the space-frequency codes obtained via the mapping by finding lower and upper bounds on their coding advantages as functions of the coding advantages of the underlying space-time codes. This result will allow us to investigate the effects of the delay distribution and the power distribution of the channel impulse responses on the performance of the resulting space-frequency codes. Extensive simulation results are also presented to illustrate and support the theory.
\end{abstract}

Index Terms-Frequency-selective fading channels, full diversity, MIMO-OFDM systems, multiple antennas, space-frequency coding, space-time coding.

\section{INTRODUCTION}

M ULTIPLE-input-multiple-output (MIMO) systems employing multiple transmit and receive antennas will inarguably play a significant role in the development of future broadband wireless communications. By taking advantage of the larger number of propagation paths between the transmit and receive antennas, the detrimental effects of channel fading can be significantly reduced. It has been shown that MIMO systems offer a large potential capacity increase compared with single antenna systems. To exploit this capacity increase, a considerable number of MIMO modulation and coding methods, which are known as space-time (ST) codes, have been proposed, for example, in [1]-[13]. The performance criteria for such transmission scenario were first derived in [1] and [2], characterizing the ST codes with two quantities: the diversity advantage, which describes the asymptotic error rate decrease as the function of the signal to noise ratio (SNR), and

Manuscript received December 13, 2002; revised April 14, 2003. The associate editor coordinating the review of this paper and approving it for publication was Prof. Brian Hughes.

W. Su, M. Olfat, and K. J. R. Liu are with the Department of Electrical and Computer Engineering and Institute for Systems Research, University of Maryland, College Park, MD 20742 USA (e-mail: weifeng@eng.umd.edu; molfat@eng.umd.edu; kjrliu@eng.umd.edu).

Z. Safar is with the Department of Innovation, IT University of Copenhagen, Copenhagen, Denmark.

Digital Object Identifier 10.1109/TSP.2003.818200 the coding advantage, which determines the vertical shift of the error performance curve.

However, most existing ST codes were developed for frequency nonselective (flat) fading channels. In case of broadband wireless communication systems, the channel exhibits frequency selectivity (delay spread), resulting in intersymbol interference (ISI) that can cause serious performance degradation. Among the various ISI mitigating approaches, orthogonal frequency division multiplexing (OFDM) is one of the most promising techniques as it eliminates the need for high complexity equalization and offers high spectral efficiency [14], [15].

In order to combine the advantages of both the MIMO systems and the OFDM, space-frequency (SF) coded MIMOOFDM systems have been proposed, ${ }^{1}$ where two-dimensional coding is applied to distribute channel symbols across space (transmit antennas) and frequency (OFDM tones). The first SF coding scheme was proposed in [17], in which previously existing ST codes were used by replacing the time domain with frequency domain. Later works [18]-[21] also described similar schemes, i.e., using ST codes directly as SF codes. The resulting SF codes could achieve only spatial diversity and were not guaranteed to achieve the full (spatial and frequency) diversity available in the MIMO frequency selective fading channels.

The performance criteria for SF-coded MIMO-OFDM systems were derived in [22]. The ultimate limits on the maximum achievable diversity order were also established. The authors showed that in general, existing ST codes cannot exploit the frequency diversity available in the frequency selective MIMO channels, and it was suggested that a completely new code design procedure will have to be developed for MIMO-OFDM systems. Later, in [23], a construction method for a class of SF codes was provided by multiplying a part of the DFT-matrix with the input symbol vectors. The obtained SF codes achieve full spatial and frequency diversity at the expense of bandwidth efficiency. For example, for a system with two transmit antennas, eight tones, and a two-ray delay profile, the coding rate is only $1 / 4$. Moreover, this approach relies on the assumption that all of the path delays are located exactly at the sampling instances of the receiver and that the power is distributed uniformly across the paths. However, in most practical situations, this is not the case.

Characterizing the performance of MIMO-OFDM systems was also considered in [24], and the maximum achievable di-

\footnotetext{
${ }^{1}$ Another coding approach is to consider ST coding directly for single-carrier frequency-selective MIMO systems (see [27], [28], and the references therein). In this paper, we follow the SF coding approach for MIMO-OFDM systems.
} 


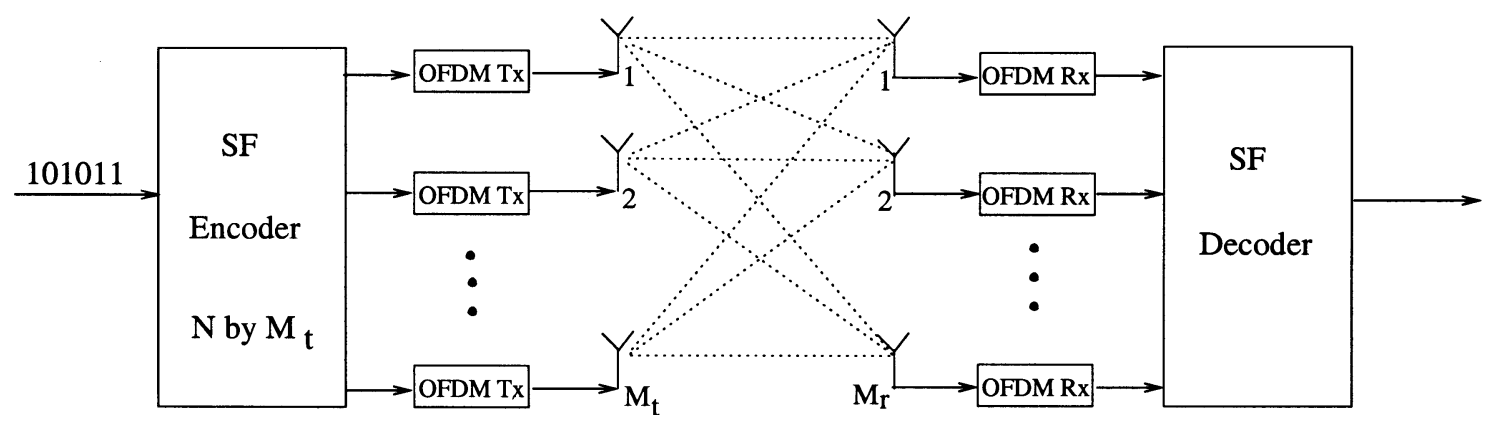

Fig. 1. SF-coded MIMO-OFDM system with $M_{t}$ transmit and $M_{r}$ receive antennas.

versity order was found to be the same as in [22]. Even though closed-form analytical expressions were derived in both [22] and [24], they do not seem useful from the viewpoint of systematic SF code design due to their complex form.

The idea of coding across multiple OFDM blocks, resulting in space-time-frequency (STF) codes, was first proposed in [25] for two transmit antennas and further developed in [26] for multiple transmit antennas. The method described in [26] provided full diversity only if the number of encoded OFDM blocks was not smaller than the number of transmit antennas. Both works assumed that the MIMO channel stays constant over multiple OFDM blocks, and the proposed STF coding schemes did not offer any additional diversity advantage compared to the SF coding approach within a single OFDM block.

In this paper, we address the problem of SF code design for MIMO-OFDM systems (i.e., coding within a single OFDM block). We propose a systematic approach to design full-diversity SF codes from ST codes for arbitrary power delay profiles. We show that ST codes and SF codes are related in the sense that ST codes achieving full (spatial) diversity in quasistatic flat fading environment can be used to construct SF codes that can achieve the maximum diversity available in frequency-selective MIMO fading channels. The relationship between the ST codes and SF codes is characterized by a simple mapping, which is independent of the particular properties of the applied ST code. Therefore, any ST code (block or trellis) designed for quasistatic flat fading channels can be used for full diversity transmission in MIMO-OFDM systems. The proposed mapping ensures full diversity at the price of symbol rate decrease compared with the symbol rate of the underlying ST codes. However, to our knowledge, the proposed SF codes have the best-known coding rate of all SF codes that are guaranteed to achieve full diversity.

We also characterize the performance of the SF codes obtained via the mapping by finding lower and upper bounds on their coding advantages as functions of the coding advantages of the underlying ST codes. This result will allow us to investigate the effects of the delay distribution and the power distribution of the channel impulse responses on the performance of the resulting SF codes.

The rest of the paper is organized as follows. In Section II, we introduce a system model, taking into account arbitrary delay profiles. In Section III, we provide an alternative formu- lation of the performance criteria for SF-coded MIMO-OFDM systems, which serves as a basis for our SF code design method. In Section IV, we describe the main contribution of this paper. We state and prove the main result: Using a simple repetition mapping, full-diversity SF codes can be constructed from any ST (block or trellis) code designed for quasistatic flat Rayleigh fading channels. In Section V, we characterize the coding advantage of the resulting SF codes in terms of the coding advantage of the underlying ST codes by defining and evaluating the diversity product for SF codes. The simulation results are presented in Section VI, and some conclusions are drawn in Section VII.

\section{SYSTEM MODEL}

We consider an SF-coded MIMO-OFDM system with $M_{t}$ transmit antennas, $M_{r}$ receive antennas, and $N$ subcarriers, as shown in Fig. 1. Suppose that the frequency-selective fading channels between each pair of transmit and receive antennas have $L$ independent delay paths and the same power delay profile. The MIMO channel is assumed to be constant over each OFDM block period. The channel impulse response from transmit antenna $i$ to receive antenna $j$ can be modeled as

$$
h_{i, j}(\tau)=\sum_{l=0}^{L-1} \alpha_{i, j}(l) \delta\left(\tau-\tau_{l}\right)
$$

where $\tau_{l}$ is the delay of the $l$ th path, and $\alpha_{i, j}(l)$ is the complex amplitude of the $l$ th path between transmit antenna $i$ and receive antenna $j$. The $\alpha_{i, j}(l)$ 's are modeled as zero-mean, complex Gaussian random variables with variances $E\left|\alpha_{i, j}(l)\right|^{2}=\delta_{l}^{2}$, where $E$ stands for the expectation. Note that the time delay $\tau_{l}$ and the variance $\delta_{l}^{2}$ are the same for each transmit-receive link [16], [20]. The powers of the $L$ paths are normalized such that $\sum_{l=0}^{L-1} \delta_{l}^{2}=1$. From (1), the frequency response of the channel is given by

$$
H_{i, j}(f)=\sum_{l=0}^{L-1} \alpha_{i, j}(l) e^{-\mathbf{j} 2 \pi f \tau_{l}}, \quad \mathbf{j}=\sqrt{-1} .
$$

We assume that the MIMO channel is spatially uncorrelated, i.e., the channel taps $\alpha_{i, j}(l)$ are independent for different indices $(i, j)$. 
The input bit stream (uncoded or coming from a channel encoder) is divided into $b$ bit-long segments, forming $2^{b}$-ary source symbols. These source symbols are parsed into blocks and mapped onto a SF codeword to be transmitted over the $M_{t}$ transmit antennas. Each SF codeword can be expressed as an $N \times M_{t}$ matrix

$$
C=\left[\begin{array}{cccc}
c_{1}(0) & c_{2}(0) & \cdots & c_{M_{t}}(0) \\
c_{1}(1) & c_{2}(1) & \cdots & c_{M_{t}}(1) \\
\vdots & \vdots & \ddots & \vdots \\
c_{1}(N-1) & c_{2}(N-1) & \cdots & c_{M_{t}}(N-1)
\end{array}\right]
$$

where $c_{i}(n)$ denotes the channel symbol transmitted over the $n$th subcarrier by transmit antenna $i$. The SF code is assumed to satisfy the energy constraint $E\|C\|_{F}^{2}=N M_{t}$, where $\|C\|_{F}$ is the Frobenius norm ${ }^{2}$ of $C$. The OFDM transmitter applies an $N$-point IFFT to each column of the matrix $C$. After appending a cyclic prefix, the OFDM symbol corresponding to the $i$ th $\left(i=1,2, \cdots, M_{t}\right)$ column of $C$ is transmitted by transmit antenna $i$. Note that all of the $M_{t}$ OFDM symbols are transmitted simultaneously from different transmit antennas.

At the receiver, after matched filtering, removing the cyclic prefix, and applying FFT, the received signal at the $n$th subcarrier at receive antenna $j$ is given by

$$
y_{j}(n)=\sqrt{\frac{\rho}{M_{t}}} \sum_{i=1}^{M_{t}} c_{i}(n) H_{i, j}(n)+z_{j}(n)
$$

where

$$
H_{i, j}(n)=\sum_{l=0}^{L-1} \alpha_{i, j}(l) e^{-\mathbf{j} 2 \pi n \Delta f \tau_{l}}
$$

is the channel frequency response at the $n$th subcarrier between transmit antenna $i$ and receive antenna $j, \Delta f=1 / T$ is the subcarrier separation in the frequency domain, and $T$ is the OFDM symbol period. We assume that the channel state information $H_{i, j}(n)$ is known at the receiver but not at the transmitter. In (4), $z_{j}(n)$ denotes the additive complex Gaussian noise with zero mean and unit variance at the $n$th subcarrier at receive antenna $j$. The noise samples $z_{j}(n)$ are assumed to be uncorrelated for different $j$ and $n$. The factor $\sqrt{\rho / M_{t}}$ in (4) ensures that $\rho$ is the average SNR at each receive antenna, independently of the number of transmit antennas.

${ }^{2}$ The Frobenius norm of $C$ is defined as

$$
\|C\|_{F}^{2}=\operatorname{tr}\left(C^{\mathcal{H}} C\right)=\operatorname{tr}\left(C C^{\mathcal{H}}\right)=\sum_{n=0}^{N-1} \sum_{i=1}^{M_{t}}\left|c_{i}(n)\right|^{2} .
$$

\section{Performance CRITERIA}

The problem of deriving performance criteria for SF-coded MIMO-OFDM systems has been addressed by several authors [22], [24]. In this section, we provide an alternative formulation based on the results of [29]-[31]. This formulation will serve as the starting point for the results to be discussed later.

The received signal (4) can be rewritten in vector form as

$$
\mathbf{Y}=\sqrt{\frac{\rho}{M_{t}}} \mathbf{D H}+\mathbf{Z}
$$

where $\mathbf{D}$ is an $N M_{r} \times N M_{t} M_{r}$ matrix constructed from the SF codeword $C$ as in (7), shown at the bottom of the page, in which

$D_{i}=\operatorname{diag}\left\{c_{i}(0), c_{i}(1), \cdots, c_{i}(N-1)\right\}, \quad i=1,2, \cdots, M_{t}$.

Each $D_{i}$ in (8) is related to the $i$ th column of the SF codeword $C$. The channel vector $\mathbf{H}$ of size $N M_{t} M_{r} \times 1$ is formatted as

$$
\begin{aligned}
\mathbf{H}=\left[H_{1,1}^{\mathcal{T}} \cdots H_{M_{t}, 1}^{\mathcal{T}} H_{1,2}^{\mathcal{T}} \cdots H_{M_{t}, 2}^{\mathcal{T}}\right. & \\
& \left.\cdots H_{1, M_{r}}^{\mathcal{T}} \cdots H_{M_{t}, M_{r}}^{\mathcal{T}}\right]^{\mathcal{T}}
\end{aligned}
$$

where

$$
H_{i, j}=\left[\begin{array}{llll}
H_{i, j}(0) & H_{i, j}(1) & \cdots & H_{i, j}(N-1)
\end{array}\right]^{\mathcal{T}} .
$$

The received signal vector $\mathbf{Y}$ of size $N M_{r} \times 1$ is given by

$$
\begin{array}{r}
\mathbf{Y}=\left[y_{1}(0) \cdots y_{1}(N-1) y_{2}(0) \cdots y_{2}(N-1)\right. \\
\left.\cdots y_{M_{r}}(0) \cdots y_{M_{r}}(N-1)\right]^{\mathcal{T}}
\end{array}
$$

and the noise vector $\mathbf{Z}$ has the same form as $\mathbf{Y}$, i.e.,

$$
\begin{array}{r}
\mathbf{Z}=\left[z_{1}(0) \cdots z_{1}(N-1) z_{2}(0) \cdots z_{2}(N-1)\right. \\
\left.\cdots z_{M_{r}}(0) \cdots z_{M_{r}}(N-1)\right]^{\mathcal{T}} .
\end{array}
$$

Suppose that $\mathbf{D}$ and $\tilde{\mathbf{D}}$ are two different matrices related to two different SF codewords $C$ and $\tilde{C}$, respectively. Then, the pairwise error probability between $\mathbf{D}$ and $\tilde{D}$ can be upper bounded as [29], [30]

$$
P(\mathbf{D} \rightarrow \tilde{\mathbf{D}}) \leq\left(\begin{array}{c}
2 r-1 \\
r
\end{array}\right)\left(\prod_{i=1}^{r} \gamma_{i}\right)^{-1}\left(\frac{\rho}{M_{t}}\right)^{-r}
$$

where $r$ is the rank of $(\mathbf{D}-\tilde{\mathbf{D}}) \mathbf{R}(\mathbf{D}-\tilde{\mathbf{D}})^{\mathcal{H}}, \gamma_{1}, \gamma_{2}, \cdots, \gamma_{r}$ are the nonzero eigenvalues of $(\mathbf{D}-\tilde{\mathbf{D}}) \mathbf{R}(\mathbf{D}-\tilde{\mathbf{D}})^{\mathcal{H}}$, and $\mathbf{R}=$ $E\left\{\mathbf{H H}^{\mathcal{H}}\right\}$ is the correlation matrix of $\mathbf{H}$. The superscript $\mathcal{H}$ stands for the complex conjugate and transpose of a matrix.

Based on the upper bound on the pairwise error probability in (13), two general SF code performance criteria can be proposed as follows.

- Diversity (rank) criterion: The minimum rank of (D $\tilde{\mathbf{D}}) \mathbf{R}(\mathbf{D}-\tilde{\mathbf{D}})^{\mathcal{H}}$ over all pairs of different codewords $C$ and $\tilde{C}$ should be as large as possible.

$$
\mathbf{D}=\left[\begin{array}{ccccccccccccc}
D_{1} & D_{2} & \cdots & D_{M} & 0 & 0 & \cdots & 0 & \cdots & 0 & 0 & \cdots & 0 \\
0 & 0 & \cdots & 0 & D_{1} & D_{2} & \cdots & D_{M} & \cdots & 0 & 0 & \cdots & 0 \\
& & \vdots & & & & \vdots & & \ddots & & & \vdots & \\
0 & 0 & \cdots & 0 & 0 & 0 & \cdots & 0 & \cdots & D_{1} & D_{2} & \cdots & D_{M}
\end{array}\right]_{N M_{r} \times N M_{t} M_{r}}
$$


- Product criterion: The minimum value of the product $\prod_{i=1}^{r} \gamma_{i}$ over all pairs of different codewords $C$ and $\tilde{C}$ should be maximized.

However, it is hard to design SF codes directly based on the discussion on $(\mathbf{D}-\tilde{\mathbf{D}}) \mathbf{R}(\mathbf{D}-\tilde{\mathbf{D}})^{\mathcal{H}}$, which is related to an $N M_{t} M_{r} \times N M_{t} M_{r}$ correlation matrix $\mathbf{R}$. In case of spatially uncorrelated MIMO channels, i.e., the channel taps $\alpha_{i, j}(l)$ are independent for different transmit antenna $i$ and receive antenna $j$, the correlation matrix $\mathbf{R}$ of size $N M_{t} M_{r} \times N M_{t} M_{r}$ becomes

$$
\begin{aligned}
\mathbf{R}= & E\left\{\mathbf{H H}^{\mathcal{H}}\right\} \\
= & \operatorname{diag}\left(R_{1,1}, \cdots, R_{M_{t}, 1}, R_{1,2}, \cdots, R_{M_{t}, 2}\right. \\
& \left.\quad \cdots, R_{1, M_{r}}, \cdots, R_{M_{t}, M_{r}}\right)
\end{aligned}
$$

where

$$
R_{i, j}=E\left\{H_{i, j} H_{i, j}^{\mathcal{H}}\right\}
$$

is the correlation matrix of the channel frequency response from transmit antenna $i$ to receive antenna $j$. Using the notation $w=$ $e^{-\mathbf{j} 2 \pi \Delta f}$, from (5) and (10), we have

$$
\begin{aligned}
H_{i, j} & =\left[\sum_{l=0}^{L-1} \alpha_{i, j}(l) \sum_{l=0}^{L-1} \alpha_{i, j}(l) w^{\tau_{l}} \cdots \sum_{l=0}^{L-1} \alpha_{i, j}(l) w^{(N-1) \tau_{l}}\right]_{(16)}^{\mathcal{T}} \\
& =W \cdot A_{i, j}
\end{aligned}
$$

where

$$
W=\left[\begin{array}{cccc}
1 & 1 & \cdots & 1 \\
w^{\tau_{0}} & w^{\tau_{1}} & \cdots & w^{\tau_{L-1}} \\
\vdots & \vdots & \ddots & \vdots \\
w^{(N-1) \tau_{0}} & w^{(N-1) \tau_{1}} & \cdots & w^{(N-1) \tau_{L-1}}
\end{array}\right]_{N \times L}
$$

and

$$
A_{i, j}=\left[\alpha_{i, j}(0) \alpha_{i, j}(1) \cdots \alpha_{i, j}(L-1)\right]^{\mathcal{T}} .
$$

Note that in general, $W$ is not a unitary matrix. If all of the $L$ delay paths fall at the sampling instances of the receiver, then $W$ is part of the DFT-matrix, which is unitary. Substituting (16) into (15), $R_{i, j}$ in (15) can be expressed as

$$
\begin{aligned}
R_{i, j} & =E\left\{W A_{i, j} A_{i, j}^{\mathcal{H}} W^{\mathcal{H}}\right\} \\
& =W E\left\{A_{i, j} A_{i, j}^{\mathcal{H}}\right\} W^{\mathcal{H}} \\
& =W \operatorname{diag}\left(\delta_{0}^{2}, \delta_{1}^{2}, \cdots, \delta_{L-1}^{2}\right) W^{\mathcal{H}} \triangleq R .
\end{aligned}
$$

The third equality follows from the assumption that the path gains $\alpha_{i, j}(l)$ are independent for different paths and different pairs of transmit and receive antennas. Note that the correlation matrix $R$ is independent of the transmit and receive antenna indices $i$ and $j$. From (14) and (17), we obtain

$$
\mathbf{R}=I_{M_{t} M_{r}} \otimes R
$$

where $\otimes$ denotes the tensor product, and $I_{M_{t} M_{r}}$ is the identity matrix of size $M_{t} M_{r} \times M_{t} M_{r}$. Therefore, combining (3), (7), (8), and (18), the expression for $(\mathbf{D}-\tilde{\mathbf{D}}) \mathbf{R}(\mathbf{D}-\tilde{\mathbf{D}})^{\mathcal{H}}$ in (13) can be rewritten as

$$
\begin{aligned}
(\mathbf{D}-\tilde{\mathbf{D}}) \mathbf{R}(\mathbf{D}-\tilde{\mathbf{D}})^{\mathcal{H}} & =I_{M_{r}} \otimes\left[\sum_{i=1}^{M_{t}}\left(D_{i}-\tilde{D}_{i}\right) R\left(D_{i}-\tilde{D}_{i}\right)^{\mathcal{H}}\right] \\
& =I_{M_{r}} \otimes\left\{\left[(C-\tilde{C})(C-\tilde{C})^{\mathcal{H}}\right] \circ R\right\}
\end{aligned}
$$

where $\circ$ denotes the Hadamard product. ${ }^{3}$ Defining the matrix $\Delta$ as

$$
\Delta \triangleq(C-\tilde{C})(C-\tilde{C})^{\mathcal{H}}
$$

and substituting (19) into (13), the pairwise error probability between $C$ and $\tilde{C}$ can be upper bounded as

$$
P(C \rightarrow \tilde{C}) \leq\left(\begin{array}{c}
2 K M_{r}-1 \\
K M_{r}
\end{array}\right)\left(\prod_{i=1}^{K} \lambda_{i}\right)^{-M_{r}}\left(\frac{\rho}{M_{t}}\right)^{-K M_{r}}
$$

where $K$ is the rank of $\Delta \circ R$, and $\lambda_{1}, \lambda_{2}, \cdots, \lambda_{K}$ are the nonzero eigenvalues of $\Delta \circ R$. As a consequence, we can formulate the performance criteria as follows.

- Diversity (rank) criterion: The minimum rank of $\Delta \circ R$ over all pairs of distinct signals $C$ and $\tilde{C}$ should be as large as possible.

- Product criterion: The minimum value of the product $\prod_{i=1}^{K} \lambda_{i}$ over all pairs of distinct signals $C$ and $\tilde{C}$ should also be maximized.

According to a rank inequality on Hadamard products [33, $\mathrm{p}$. 307], we have the relationship

$$
\operatorname{rank}(\Delta \circ R) \leq \operatorname{rank}(\Delta) \operatorname{rank}(R) .
$$

Since the rank of $\Delta$ is at most $M_{t}$, the rank of $R$ is at most $L$, and the rank of $\Delta \circ R$ is at most $N$, we obtain

$$
\operatorname{rank}(\Delta \circ R) \leq \min \left\{L M_{t}, N\right\} .
$$

Thus, the maximum achievable diversity is at most $\min \left\{L M_{t} M_{r}, N M_{r}\right\}$, which is in agreement with the results of [22], [24] and [25].

\section{ACHIEVING Full Diversity VIA MAPPING}

In this section, we propose an approach to systematically design full diversity SF codes from ST codes. We show that using a simple repetition mapping, full-diversity SF codes can be constructed from any ST (block or trellis) code designed for quasistatic flat Rayleigh fading channels.

In the sequel, the SF encoder will consist of a ST encoder and a mapping $\mathcal{M}_{l}$, as shown in Fig. 2. For each $1 \times M_{t}$ output vector $\left(g_{1} g_{2} \cdots g_{M_{t}}\right)$ from the ST encoder and a fixed number $l(1 \leq l \leq L)$, the mapping $\mathcal{M}_{l}$ is defined as

$$
\mathcal{M}_{l}:\left(g_{1} g_{2} \cdots g_{M_{t}}\right) \rightarrow \mathbf{1}_{l \times 1}\left(g_{1} g_{2} \cdots g_{M_{t}}\right)
$$

where $1_{l \times 1}$ is an all one matrix of size $l \times 1$. The resulting $l \times M_{t}$ matrix is actually a repetition of the vector $\left(g_{1} g_{2} \cdots g_{M_{t}}\right) l$ times. Suppose that $l M_{t}$ is not greater than the number of OFDM subcarriers $N$, and $k$ is the largest integer such that $k l M_{t} \leq N$. Denote the output code matrix of the ST encoder by $G$. (For a space-time block encoder, $G$ is a concatenation of some block codewords. For a space-time trellis encoder, $G$ corresponds to a path of length $k M_{t}$ starting and ending at the

${ }^{3}$ Suppose that $A=\left\{a_{i, j}\right\}$ and $B=\left\{b_{i, j}\right\}$ are two matrices of size $m \times n$. The Hadamard product of $A$ and $B$ is defined as

$$
A \circ B=\left[\begin{array}{ccc}
a_{1,1} b_{1,1} & \cdots & a_{1, n} b_{1, n} \\
\cdots & \cdots & \cdots \\
a_{m, 1} b_{m, 1} & \cdots & a_{m, n} b_{m, n}
\end{array}\right] .
$$




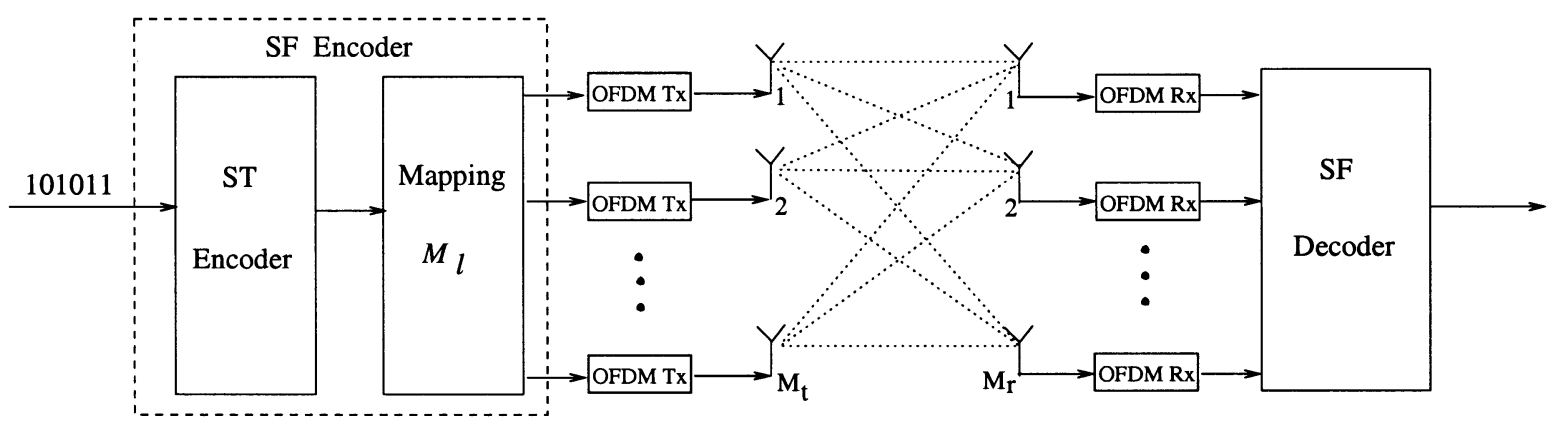

Fig. 2. SF encoder consisting of an ST encoder and a mapping.

zero state.) Then, the $\mathrm{SF}$ code $C$ of size $N \times M_{t}$ is constructed as

$$
C=\left[\begin{array}{c}
\mathcal{M}_{l}(G) \\
\mathbf{0}_{\left(N-k l M_{t}\right) \times M_{t}}
\end{array}\right]
$$

where

$$
\mathcal{M}_{l}(G)=\left[I_{k} M_{t} \otimes \mathbf{1}_{l \times 1}\right] G .
$$

In fact, the SF code $C$ is obtained by repeating each row of $G l$ times and adding some zeros. The zero padding used here ensures that the space-frequency code $C$ has size $N \times M_{t}$. Typically, the size of the zero padding is small, and it can be used to drive the trellis encoder to the zero state. The following theorem states that if the employed ST code $G$ has full diversity for quasistatic flat fading channels, the space-frequency code constructed by (24) will achieve a diversity of at least $l M_{t} M_{r}$.

Theorem 1: Suppose that an MIMO-OFDM system equipped with $M_{t}$ transmit and $M_{r}$ receive antennas has $N$ subcarriers and that the frequency-selective channel has $L$ independent paths, in which the maximum path delay is less than one OFDM block period. If an ST (block or trellis) code designed for $M_{t}$ transmit antennas achieves full diversity for quasistatic flat fading channels, then the SF code obtained from this ST code via the mapping $\mathcal{M}_{l}(1 \leq l \leq L)$ defined in (25) will achieve a diversity order of at least $\min \left\{l M_{t} M_{r}, N M_{r}\right\}$.

Proof: Since in typical MIMO-OFDM systems the number of subcarriers $N$ is greater than $L M_{t}$, we provide the proof for the $l M_{t} \leq N$ case for a given mapping $\mathcal{M}_{l}$, $1 \leq l \leq L$. If $l M_{t}>N$, the proof is similar to the one described below and is omitted for brevity. Assume that $k$ is the largest integer such that $k l M_{t} \leq N$.

For two distinct SF codewords $C$ and $\tilde{C}$ of size $N \times M_{t}$, there are two corresponding distinct ST codewords $G$ and $\tilde{G}$ of size $k M_{t} \times M_{t}$ such that

$$
C-\tilde{C}=\left[\begin{array}{c}
\mathcal{M}_{l}(G-\tilde{G}) \\
\mathbf{0}_{\left(N-k l M_{t}\right) \times M_{t}}
\end{array}\right]
$$

in which

$$
\mathcal{M}_{l}(G-\tilde{G})=\left[I_{k M_{t}} \otimes \mathbf{1}_{l \times 1}\right](G-\tilde{G}) .
$$

Since the ST code achieves full diversity for quasistatic flat fading channels, $G-\tilde{G}$ is of full rank for two distinct $G$ and $\tilde{G}$, i.e. the rank of $G-\tilde{G}$ is $M_{t}$.
Using the performance criteria described in Section III, the objective of the proof is to show that the matrix $\Delta \circ R$ has a rank of at least $l M_{t}$. From (20) and (26), we have

$$
\Delta=\left[\begin{array}{cc}
\mathcal{M}_{l}(G-\tilde{G})\left[\mathcal{M}_{l}(G-\tilde{G})\right]^{\mathcal{H}} & \mathbf{0}_{k l M_{t} \times\left(N-k l M_{t}\right)} \\
\mathbf{0}_{\left(N-k l M_{t}\right) \times k l M_{t}} & \mathbf{0}_{\left(N-k l M_{t}\right) \times\left(N-k l M_{t}\right)}
\end{array}\right] .
$$

Thus, in $\Delta \circ R$, all entries are zero, except for a $k l M_{t} \times k l M_{t}$ submatrix. Denote this $k l M_{t} \times k l M_{t}$ submatrix as $(\Delta \circ R)_{k l M_{t} \times k l M_{t}}$.

On the other hand, from (17), it can be verified that the entries of the correlation matrix $R=\left\{r_{i, j}\right\}_{1 \leq i, j \leq N}$ can be expressed as

$$
r_{i, j}=\sum_{s=0}^{L-1} \delta_{s}^{2} w^{(i-j) \tau_{s}}, \quad 1 \leq i, j \leq N .
$$

Therefore, from (26)-(28), we obtain

$$
\begin{aligned}
&(\Delta \circ R)_{k l M_{t} \times k l M_{t}}=\left\{\mathcal{M}_{l}(G-\tilde{G})\left[\mathcal{M}_{l}(G-\tilde{G})\right]^{\mathcal{H}}\right\} \circ P \\
&=\left\{\left[I_{k M_{t}} \otimes \mathbf{1}_{l \times 1}\right](G-\tilde{G})(G-\tilde{G})^{\mathcal{H}}\right. \\
&\left.\times\left[I_{k M_{t}} \otimes \mathbf{1}_{l \times 1}\right]^{\mathcal{H}}\right\} \circ P \\
&=\{[ {\left[(G-\tilde{G})(G-\tilde{G})^{\mathcal{H}}\right] } \\
&\left.\otimes \mathbf{1}_{l \times l}\right\} \circ P
\end{aligned}
$$

where $P=\left\{p_{i, j}\right\}_{1 \leq i, j \leq k l M_{t}}$ is a $k l M_{t} \times k l M_{t}$ matrix with entries

$$
p_{i, j}=\sum_{s=0}^{L-1} \delta_{s}^{2} w^{(i-j) \tau_{s}}, \quad 1 \leq i, j \leq k l M_{t} .
$$

The last equality in (29) follows from the identities $\left[I_{k M_{t}} \otimes\right.$ $\left.\mathbf{1}_{l \times 1}\right]^{\mathcal{H}}=I_{k M_{t}} \otimes \mathbf{1}_{1 \times l}$ and $\left(A_{1} \otimes B_{1}\right)\left(A_{2} \otimes B_{2}\right)\left(A_{3} \otimes B_{3}\right)=$ $\left(A_{1} A_{2} A_{3}\right) \otimes\left(B_{1} B_{2} B_{3}\right)[33$, p. 251].

We further partition the $k l M_{t} \times k l M_{t}$ matrix $P$ into $l \times l$ submatrices as follows:

$$
P=\left[\begin{array}{cccc}
P_{1,1} & P_{1,2} & \cdots & P_{1, k M_{t}} \\
P_{2,1} & P_{2,2} & \cdots & P_{2, k M_{t}} \\
\vdots & \vdots & \ddots & \vdots \\
P_{k M_{t}, 1} & P_{k M_{t}, 2} & \cdots & P_{k M_{t}, k M_{t}}
\end{array}\right]
$$


where each submatrix $P_{m, n}, 1 \leq m, n \leq k M_{t}$ is of size $l \times l$. Denoting the entries of $P_{m, n}$ as $p_{m, n}(i, j), 1 \leq i, j \leq l$, we obtain

$$
p_{m, n}(i, j)=\sum_{s=0}^{L-1} \delta_{s}^{2} w^{[(m-n) l+(i-j)] \tau_{s}}, \quad 1 \leq i, j \leq l .
$$

As a consequence, each submatrix $P_{m, n}, 1 \leq m, n \leq k M_{t}$, can be expressed as

$$
P_{m, n}=W_{m} \operatorname{diag}\left\{\delta_{0}^{2}, \delta_{1}^{2}, \cdots, \delta_{L-1}^{2}\right\} W_{n}^{\mathcal{H}}
$$

where $W_{m}$ is specified in (34), shown at the bottom of the page, for $m=1,2, \cdots, k M_{t}$. In (34), $W_{m}$ can be further decomposed as

$$
W_{m}=W_{1} \operatorname{diag}\left\{w^{(m-1) l \tau_{0}}, w^{(m-1) l \tau_{1}}, \cdots, w^{(m-1) l \tau_{L-1}}\right\}
$$

for $1 \leq m \leq k M_{t}$, where

$$
W_{1}=\left[\begin{array}{cccc}
1 & 1 & \cdots & 1 \\
w^{\tau_{0}} & w^{\tau_{1}} & \cdots & w^{\tau_{L-1}} \\
\vdots & \vdots & \ddots & \vdots \\
w^{(l-1) \tau_{0}} & w^{(l-1) \tau_{1}} & \cdots & w^{(l-1) \tau_{L-1}}
\end{array}\right] .
$$

Let us denote the matrix consisting of the first $l$ columns of $W_{1}$ by $W_{0}$. We observe that $W_{0}$ is an $l \times l$ Vandermonde matrix in $l$ variables $w^{\tau_{0}}, w^{\tau_{1}}, \cdots, w^{\tau_{l-1}}[33$, p. 400]. The determinant of $W_{0}$ can be calculated as follows:

$$
\begin{aligned}
\operatorname{det}\left(W_{0}\right) & =\prod_{0 \leq i<j \leq l-1}\left(w^{\tau_{j}}-w^{\tau_{i}}\right) \\
& =\prod_{0 \leq i<j \leq l-1}\left[e^{-\mathbf{j} 2 \pi \Delta f\left(\tau_{j}-\tau_{i}\right)}-1\right] e^{-\mathbf{j} 2 \pi \Delta f \tau_{i}} .
\end{aligned}
$$

Since $\Delta f$ is the inverse of the OFDM block period $T$ and the maximum path delay is less than $T$, we have $\Delta f\left(\tau_{j}-\tau_{i}\right)<1$ for any $0 \leq i<j \leq l-1$. Thus, $W_{0}$ is of full rank, and so is $W_{1}$. It follows that for any $m=1,2, \cdots, k M_{t}$, the rank of $W_{m}$ is $l$.

We now go back to (29) to investigate the rank of $\Delta \circ R$. For convenience, we use the notation

$$
(G-\tilde{G})(G-\tilde{G})^{\mathcal{H}} \triangleq\left[\begin{array}{cccc}
a_{1,1} & a_{1,2} & \cdots & a_{1, k M_{t}} \\
a_{2,1} & a_{2,2} & \cdots & a_{2, k M_{t}} \\
\vdots & \vdots & \ddots & \vdots \\
a_{k M_{t}, 1} & a_{k M_{t}, 2} & \cdots & a_{k M_{t}, k M_{t}}
\end{array}\right]
$$

and $\Lambda=\operatorname{diag}\left\{\delta_{0}^{2}, \delta_{1}^{2}, \cdots, \delta_{L-1}^{2}\right\}$. Then, substituting (31) and (33) into (29), we obtain (37), shown at the bottom of the page.

Since the rank of $G-\tilde{G}$ is $M_{t}$, there are $M_{t}$ linearly independent rows in $G-\tilde{G}$. Suppose that the $f_{i}$ th, $1 \leq f_{1}<f_{2}<$ $\cdots<f_{M_{t}} \leq k M_{t}$, rows of $G-\tilde{G}$ are linearly independent of each other. Then, the matrix

$$
A \triangleq\left[\begin{array}{cccc}
a_{f_{1}, f_{1}} & a_{f_{1}, f_{2}} & \cdots & a_{f_{1}, f_{M_{t}}} \\
a_{f_{2}, f_{1}} & a_{f_{2}, f_{2}} & \cdots & a_{f_{2}, f_{M_{t}}} \\
\vdots & \vdots & \ddots & \vdots \\
a_{f_{M_{t}}, f_{1}} & a_{f_{M_{t}}, f_{2}} & \cdots & a_{f_{M_{t}}, f_{M_{t}}}
\end{array}\right]
$$

is a submatrix of $(G-\tilde{G})(G-\tilde{G})^{\mathcal{H}}$, and the rank of $A$ is $M_{t}$. Using the notation

$$
Q_{0}=\operatorname{diag}\left\{W_{f_{1}}, W_{f_{2}}, \cdots, W_{f_{M_{t}}}\right\}
$$

from (37), we can see that $Q_{0}\{A \otimes \Lambda\} Q_{0}^{\mathcal{H}}$ is an $l M_{t} \times l M_{t}$ submatrix of $(\Delta \circ R)_{k l M_{t} \times k l M_{t}}$. Therefore, to show that the

$$
W_{m}=\left[\begin{array}{cccc}
w^{(m-1) l \tau_{0}} & w^{(m-1) l \tau_{1}} & \cdots & w^{(m-1) l \tau_{L-1}} \\
w^{[(m-1) l+1] \tau_{0}} & w^{[(m-1) l+1] \tau_{1}} & \cdots & w^{[(m-1) l+1] \tau_{L-1}} \\
\vdots & \vdots & \ddots & \vdots \\
w^{[(m-1) l+(l-1)] \tau_{0}} & w^{[(m-1) l+(l-1)] \tau_{1}} & \cdots & w^{[(m-1) l+(l-1)] \tau_{L-1}}
\end{array}\right]
$$

$$
\begin{aligned}
(\Delta & \circ R)_{k l M_{t} \times k l M_{t}} \\
= & {\left[\begin{array}{cccc}
a_{1,1} P_{1,1} & a_{1,2} P_{1,2} & \ldots & a_{1, k M_{t}} P_{1, k M_{t}} \\
a_{2,1} P_{2,1} & a_{2,2} P_{2,2} & \ldots & a_{2, k M_{t}} P_{2, k M_{t}} \\
\vdots & \vdots & \ddots & \vdots \\
a_{k M_{t}, 1} P_{k M_{t}, 1} & a_{k M_{t}, 2} P_{k M_{t}, 2} & \cdots & a_{k M_{t}, k M_{t}} P_{k M_{t}, k M_{t}}
\end{array}\right] } \\
= & {\left[\begin{array}{cccc}
W_{1} a_{1,1} \Lambda W_{1}^{\mathcal{H}} & W_{1} a_{1,2} \Lambda W_{2}^{\mathcal{H}} & \cdots & W_{1} a_{1, k M_{t}} \Lambda W_{k M_{t}}^{\mathcal{H}} \\
W_{2} a_{2,1} \Lambda W_{1}^{\mathcal{H}} & W_{2} a_{2,2} \Lambda W_{2}^{\mathcal{H}} & \ldots & W_{2} a_{2, k M_{t}} \Lambda W_{k M_{t}}^{\mathcal{H}} \\
\vdots & \vdots & \ddots & \vdots \\
W_{k M_{t}} a_{k M_{t}, 1} \Lambda W_{1}^{\mathcal{H}} & W_{k M_{t}} a_{k M_{t}, 2} \Lambda W_{2}^{\mathcal{H}} & \cdots & W_{k M_{t}} a_{k M_{t}, k M_{t}} \Lambda W_{k M_{t}}^{\mathcal{H}}
\end{array}\right] } \\
= & Q\left\{\left[(G-\tilde{G})(G-\tilde{G})^{\mathcal{H}}\right] \otimes \Lambda\right\} Q^{\mathcal{H}}
\end{aligned}
$$

where

$$
Q=\operatorname{diag}\left\{W_{1}, W_{2}, \cdots, W_{k M_{t}}\right\} .
$$


rank of $\Delta \circ R$ is at least $l M_{t}$, it is sufficient to show that the submatrix $Q_{0}\{A \otimes \Lambda\} Q_{0}^{\mathcal{H}}$ has rank $l M_{t}$.

Since the rank of $A$ is $M_{t}$ and the rank of $\Lambda$ is $L$, according to a rank equality on tensor products [33, p. 246], we have

$$
\operatorname{rank}(A \otimes \Lambda)=\operatorname{rank}(A) \operatorname{rank}(\Lambda)=M_{t} L
$$

so the matrix $A \otimes \Lambda$ is of full rank. Recall that for any $m=$ $1,2, \cdots, k M_{t}$, the rank of $W_{m}$ is $l$, so the rank of $Q_{0}$ is $l M_{t}$. Therefore, the rank of $Q_{0}\{A \otimes \Lambda\} Q_{0}^{\mathcal{H}}$ is $l M_{t}$. This proves the theorem.

In addition, from the proof of Theorem 1, we can see that the SF code obtained from a space-time block code of square size via the mapping $\mathcal{M}_{l}(1 \leq l \leq L)$ will achieve a diversity of $l M_{t} M_{r}$ exactly. Since the maximum achievable diversity is upper bounded by $\min \left\{L M_{t} M_{r}, N M_{r}\right\}$ [see (22)], we arrive at the following result.

Corollary 1: Under the assumptions of Theorem 1, the SF code obtained from a full diversity ST code via the mapping $\mathcal{M}_{L}$ defined in (25) achieves the maximum achievable diversity $\min \left\{L M_{t} M_{r}, N M_{r}\right\}$.

The symbol rate of the resulting SF codes obtained via the mapping $\mathcal{M}_{l}(25)$ is $1 / l$ times that of the corresponding ST codes. However, to our knowledge, the proposed SF codes have the best known symbol rate of all SF codes that are guaranteed to achieve full diversity. For example, for a system with two transmit antennas, eight subcarriers, and a two-ray delay profile, the symbol rate of the full-diversity SF codes introduced here is $1 / 2$, whereas the symbol rate in [23] is only $1 / 4$. In certain practical situations, this effect can be compensated by expanding the constellation size, maintaining the same spectral efficiency. Furthermore, from a system performance point of view, there is a tradeoff between the diversity order and the coding rate. Theorem 1 offers a flexible choice on the diversity order.

\section{Coding Advantage}

In this section, we characterize the coding advantage of the resulting SF codes in terms of the coding advantage of the underlying ST codes by defining and evaluating the diversity product for SF codes. We also analyze the effect of the delay distribution and the power distribution on the performance of the proposed SF codes.

The diversity product, which is the normalized coding advantage of a full diversity ST code, has been defined for quasistatic flat-fading channels as [8], [9], [31]

$$
\zeta_{\mathrm{ST}}=\frac{1}{2 \sqrt{M_{t}}} \min _{G \neq \tilde{G}}\left|\prod_{i=1}^{M_{t}} \beta_{i}\right|^{\frac{1}{2 M_{t}}}
$$

where $\beta_{1}, \beta_{2}, \cdots, \beta_{M_{t}}$ are the nonzero eigenvalues of $(G-$ $\tilde{G})(G-\tilde{G})^{\mathcal{H}}$ for any pair of distinct ST codewords $G$ and $\tilde{G}$.

Based on the upper bound on the pair-wise error probability (21), the diversity product of a full-diversity SF code can be defined as

$$
\zeta_{\mathrm{SF}, R}=\frac{1}{2 \sqrt{M_{t}}} \min _{C \neq \tilde{C}}\left|\prod_{i=1}^{L M_{t}} \lambda_{i}\right|^{\frac{1}{2 L M_{t}}}
$$

where $\lambda_{1}, \lambda_{2}, \cdots, \lambda_{L M_{t}}$ are the nonzero eigenvalues of $\Delta \circ R$ for any pair of distinct space-frequency codewords $C$ and $\tilde{C}$. In the rest of this section, without loss of generality, we assume that the number of subcarriers $N$ is not less than $L M_{t}$, i.e., $L M_{t} \leq N$.

The relationship between the diversity products of the full diversity SF codes obtained via the repetition mapping and the underlying ST codes is characterized by the following theorem.

Theorem 2: The diversity product of the full-diversity SF code in Corollary 1 is bounded by that of the corresponding ST code as follows:

$$
\sqrt{\eta_{L}} \Phi \zeta_{\mathrm{ST}} \leq \zeta_{\mathrm{SF}, R} \leq \sqrt{\eta_{1}} \Phi \zeta_{\mathrm{ST}}
$$

where $\Phi=\left(\prod_{l=0}^{L-1} \delta_{l}\right)^{1 / L}$, and $\eta_{1}$ and $\eta_{L}$ are the largest and smallest eigenvalues, respectively, of the matrix $H$, which is defined as

$$
H=\left[\begin{array}{cccc}
H(0) & H(1)^{*} & \cdots & H(L-1)^{*} \\
H(1) & H(0) & \cdots & H(L-2)^{*} \\
\vdots & \vdots & \ddots & \vdots \\
H(L-1) & H(L-2) & \cdots & H(0)
\end{array}\right]_{L \times L}
$$

and the entries of $H$ are given by

$$
H(n)=\sum_{l=0}^{L-1} e^{-\mathbf{j} 2 \pi n \Delta f \tau_{l}}, \quad n=0,1, \cdots, L-1 .
$$

Proof: In the following, we use the notation developed in the proof of Theorem 1 by replacing the repetition factor $l$ as $L$, since the full diversity is achieved in Corollary 1 by using the mapping $\mathcal{M}_{L}$. For any $n \times n$ non-negative definite matrix $A$, we denote its eigenvalues in a nonincreasing order as $\operatorname{eig}_{1}(A) \geq$ $\operatorname{eig}_{2}(A) \geq \cdots \geq \operatorname{eig}_{n}(A)$.

For two distinct SF codewords $C$ and $\tilde{C}$, there are two corresponding ST codewords $G$ and $\tilde{G}$ such that the relationship of $C-\tilde{C}$ and $G-\tilde{G}$ in (26) and (27) holds. According to (22) and Corollary 1 , the rank of $\Delta \circ R$ is exactly $L M_{t}$, which means that $\Delta \circ R$ has totally $L M_{t}$ nonzero eigenvalues, which are the same as the nonzero eigenvalues of $(\Delta \circ R)_{k L M_{t} \times k L M_{t}}$. Thus

$$
\begin{aligned}
\zeta_{\mathrm{SF}, R}=\frac{1}{2 \sqrt{M_{t}}} \min _{C \neq \tilde{C}} \mid \prod_{i=1}^{L M_{t}} \operatorname{eig}_{i}\left(\left.(\Delta \circ R)_{\left.k L M_{t} \times k L M_{t}\right)}\right|^{\frac{1}{2 L M_{t}}}\right. \\
=\frac{1}{2 \sqrt{M_{t}}} \min _{G \neq \tilde{G}} \mid \prod_{i=1}^{L M_{t}} \operatorname{eig}_{i}\left(Q \left\{\left[(G-\tilde{G})(G-\tilde{G})^{\mathcal{H}}\right]\right.\right. \\
=\frac{1}{2 \sqrt{M_{t}}} \min _{G \neq \tilde{G}} \mid \prod_{i=1}^{L M_{t}} \theta_{i} \operatorname{eig}_{i}\left(\left[(G-\tilde{G})(G-\tilde{G})^{\mathcal{H}}\right]\right. \\
\otimes \Lambda)\left.\right|^{\frac{1}{2 L M_{t}}}
\end{aligned}
$$

where $\operatorname{eig}_{k L M_{t}}\left(Q Q^{\mathcal{H}}\right) \leq \theta_{i} \leq \operatorname{eig}_{1}\left(Q Q^{\mathcal{H}}\right)$ for $i=1,2, \cdots$, $L M_{t}$. In (42), the second equality follows from (37), and the 
last equality follows by Ostrowski's theorem [32, p. 224]. Since $Q=\operatorname{diag}\left\{W_{1}, W_{2}, \cdots, W_{k M_{t}}\right\}$, we have

$$
Q Q^{\mathcal{H}}=\operatorname{diag}\left\{W_{1} W_{1}^{\mathcal{H}}, W_{2} W_{2}^{\mathcal{H}}, \cdots, W_{k M_{t}} W_{k M_{t}}^{\mathcal{H}}\right\} .
$$

As a requirement of Ostrowski's theorem, the matrix $Q$ should be nonsingular, which is guaranteed by the fact that each matrix $W_{m}$ is of full rank for any $m=1,2, \cdots, k M_{t}$. Furthermore, from (35), we know that for any $1 \leq m \leq k M_{t}$

$$
W_{m} W_{m}^{\mathcal{H}}=W_{1} D D^{\mathcal{H}} W_{1}^{\mathcal{H}}=W_{1} W_{1}^{\mathcal{H}}
$$

where $D=\operatorname{diag}\left\{w^{(m-1) L \tau_{0}}, w^{(m-1) L \tau_{1}} \cdots, w^{(m-1) L \tau_{L-1}}\right\}$. From (36), it is easy to verify that $W_{1} W_{1}^{\mathcal{H}}$ is the matrix $H$ defined in (41). Thus, $Q Q^{\mathcal{H}}=I_{k M_{t}} \otimes H$. Therefore, we can conclude that $\operatorname{eig}_{L}(H) \leq \theta_{i} \leq \operatorname{eig}_{1}(H)$ for any $i=1,2, \cdots, L M_{t}$.

Since the set of $L M_{t}$ nonzero eigenvalues of $[(G-\tilde{G})(G-$ $\left.\tilde{G})^{\mathcal{H}}\right] \otimes \Lambda$ can be expressed as [32, p. 246]

$$
\left\{\operatorname{eig}_{i}\left((G-\tilde{G})(G-\tilde{G})^{\mathcal{H}}\right) \cdot \operatorname{eig}_{j}(\Lambda): 1 \leq i \leq M_{t}, 1 \leq j \leq L\right\}
$$

substituting (43) into (42), we arrive at

$$
\begin{aligned}
\zeta_{\mathrm{SF}, R}= & \frac{1}{2 \sqrt{M_{t}}} \min _{G \neq \tilde{G}}\left(\prod_{i=1}^{L M_{t}} \theta_{i}\right)^{\frac{1}{2 L M_{t}}} \\
& \times\left|\prod_{i=1}^{M_{t}} \operatorname{eig}_{i}\left((G-\tilde{G})(G-\tilde{G})^{\mathcal{H}}\right)\right|^{\frac{1}{2 M_{t}}}\left(\prod_{j=1}^{L} \operatorname{eig}_{j}(\Lambda)\right)^{\frac{1}{2 L}} \\
= & \left(\prod_{i=1}^{L M_{t}} \theta_{i}\right)^{\frac{1}{2 L M_{t}}}\left(\prod_{j=1}^{L} \delta_{j}^{2}\right)^{\frac{1}{2 L}} \zeta_{\mathrm{ST}} .
\end{aligned}
$$

Since $\eta_{L} \leq \theta_{i} \leq \eta_{1}$ for any $i=1,2, \cdots, L M_{t}$, we have the inequalities in (40).

From Theorem 2, we can see that the larger the coding advantage of the ST code, the larger the coding advantage of the resulting SF code, suggesting that to maximize the performance of the SF codes, we should look for the best known ST codes existing in the literature. Moreover, the coding advantage of the SF code depends on the power delay profile. First, it depends on the power distribution through the square root of the geometric average of path powers, i.e., $\Phi=\left(\prod_{l=0}^{L-1} \delta_{l}\right)^{1 / L}$. Since the sum of the powers of the paths is unity, this implies that the best performance is expected in case of uniform power distribution (i.e. $\left.\delta_{l}^{2}=1 / L\right)$. Second, the entries of the matrix $H$ defined in (41) are functions of the path delays, and therefore, the coding advantage also depends on the delay distribution of the paths. For example, in case of a two-ray delay profile (i.e., $L=2$ ), the matrix $H$ in (41) has two eigenvalues:

$$
\begin{aligned}
& \eta_{1}=2+2\left|\cos \frac{\pi\left(\tau_{1}-\tau_{0}\right)}{T}\right| \\
& \eta_{2}=2-2\left|\cos \frac{\pi\left(\tau_{1}-\tau_{0}\right)}{T}\right| .
\end{aligned}
$$

Typically, the ratio of $\left(\tau_{1}-\tau_{0}\right) / T$ is less than $1 / 2$; therefore, $\cos \pi\left(\tau_{1}-\tau_{0}\right) / T$ is non-negative. Thus, the smaller the separation of the two rays, the smaller the eigenvalue $\eta_{2}$. If the two rays are very close compared with the duration of one OFDM symbol $T$, the lower bound in (40) approaches zero. The simulation results seem to suggest that the behavior of the coding advantage is close to the lower bound.

\section{Simulation RESUlts}

To illustrate the above analytical results, we performed some computer simulations for SF codes constructed from both ST block codes and trellis codes. The simulated SF block codes were obtained from orthogonal ST block codes for two and four transmit antennas. For two transmit antennas, the used $2 \times 2$ orthogonal ST block code was Alamouti's structure [3], which is given by

$$
G_{2}=\left[\begin{array}{cc}
x_{1} & x_{2} \\
-x_{2}^{*} & x_{1}^{*}
\end{array}\right] .
$$

The SF block code for four transmit antennas was obtained from the $4 \times 4$ orthogonal design (see [10] and the references therein)

$$
G_{4}=\left[\begin{array}{cccc}
x_{1} & x_{2} & x_{3} & 0 \\
-x_{2}^{*} & x_{1}^{*} & 0 & x_{3} \\
-x_{3}^{*} & 0 & x_{1}^{*} & -x_{2} \\
0 & -x_{3}^{*} & x_{2}^{*} & x_{1}
\end{array}\right] .
$$

In both cases, the $x_{i}$ s were taken from BPSK or QPSK constellations. Note that the $2 \times 2$ orthogonal design could carry one channel symbol per subcarrier, while the $4 \times 4$ block code had a symbol rate of only $3 / 4$. For SF trellis codes, we used the two-antenna, four-state, QPSK space-time code, and the three-antenna, 16-state, QPSK space-time code described in [11].

We simulated the SF block codes and trellis codes with different power delay profiles: a two-ray equal power delay profile and COST207 typical urban six-ray power delay profile. The subcarrier path gains were generated according to (5), independently for different transmit and receive antennas. We present average bit error rate (BER) curves as functions of the average SNR per bit. Note that in our simulations for SF block codes, we compare the proposed full-diversity SF block codes with the SF codes using ST codes directly at a same spectral efficiency.

\section{A. Simulations With Two-Ray Delay Profiles}

First, we assumed a simple two-ray, equal-power delay profile, with a delay of $\tau \mu s$ between the two rays. We simulated two cases: i) $\tau=5 \mu \mathrm{s}$ and ii) $\tau=20 \mu \mathrm{s}$. The simulated communication system had $N=128$ subcarriers, and the total bandwidth was $B W=1 \mathrm{MHz}$. Thus, the OFDM block duration was $T=128 \mu s$ without the cyclic prefix. We set the length of the cyclic prefix to $20 \mu \mathrm{s}$ for all cases. The MIMO-OFDM systems had one receive antenna. In all simulation curves, the dashed lines correspond to the $\tau=5 \mu \mathrm{s}$ case, and the solid lines correspond to the $\tau=20 \mu \mathrm{s}$ case. The curves with squares (" $\square$ ") show the performance of the full-diversity SF codes obtained by repeating each row of the ST codes two times. The curves with circles ("O") show the results for the case of using ST codes directly as SF codes (i.e. no repetition).

Fig. 3 depicts the performance of the SF block codes obtained from the two-antenna orthogonal design. We used BPSK modulation for the nonrepeated case and QPSK for the repeated case. Therefore, both systems had a spectral efficiency of $((128 \times$ 


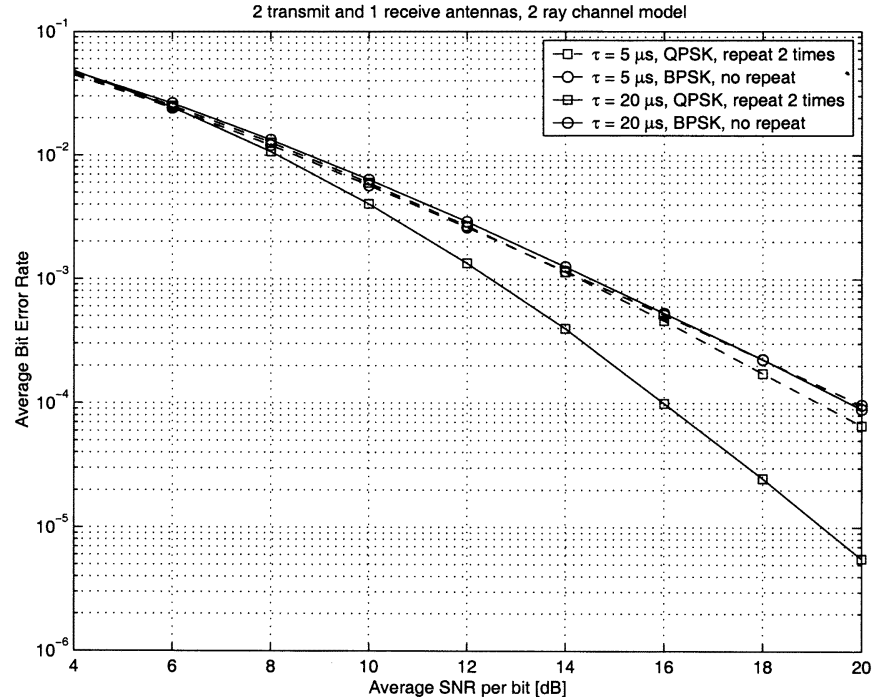

Fig. 3. Performance of two-antenna SF block codes with the two-ray channel model.

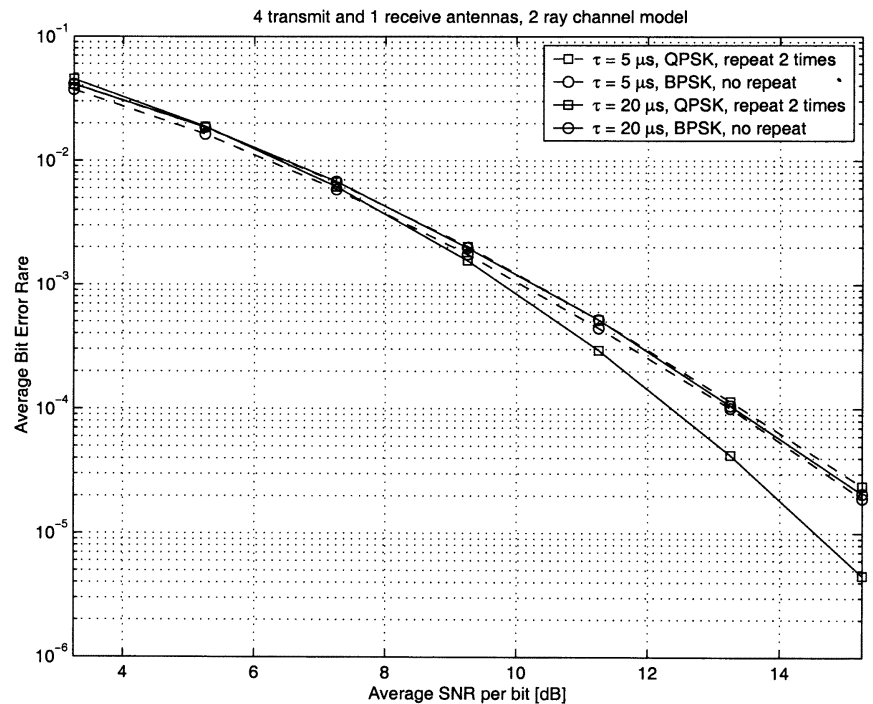

Fig. 4. Performance of four-antenna SF block codes with the two-ray channel model.

$1 \mathrm{~b}) /((128 \mu \mathrm{s}+20 \mu \mathrm{s}) \times 1 \mathrm{MHz}))=0.86 \mathrm{~b} / \mathrm{s} / \mathrm{Hz}$. The figure shows that in case of $\tau=20 \mu \mathrm{s}$, the performance curve of the full-diversity SF code has a steeper slope than that of the code without repetition. We can observe a performance improvement of about $4 \mathrm{~dB}$ at a BER of $10^{-4}$. The performance of the full-diversity SF code degraded significantly from the $\tau=20 \mu$ s case to the $\tau=5 \mu$ s case, whereas the performance of the SF code using ST code without repetition was almost the same for the two delay profiles. This observation is consistent with the theoretical result in Section $\mathrm{V}$ that the coding advantage depends on the delay distribution of the multiple paths. It is also in agreement with the result in [22] that using ST codes directly as SF codes can exploit only the spatial diversity and cannot exploit the frequency diversity. Fig. 4 shows the performance of the SF block codes obtained from the orthogonal ST code for four transmit antennas. The full-diversity SF code with repetition used QPSK modulation, and the nonrepeated code used BPSK modulation. Thus, the spectral efficiency of both codes

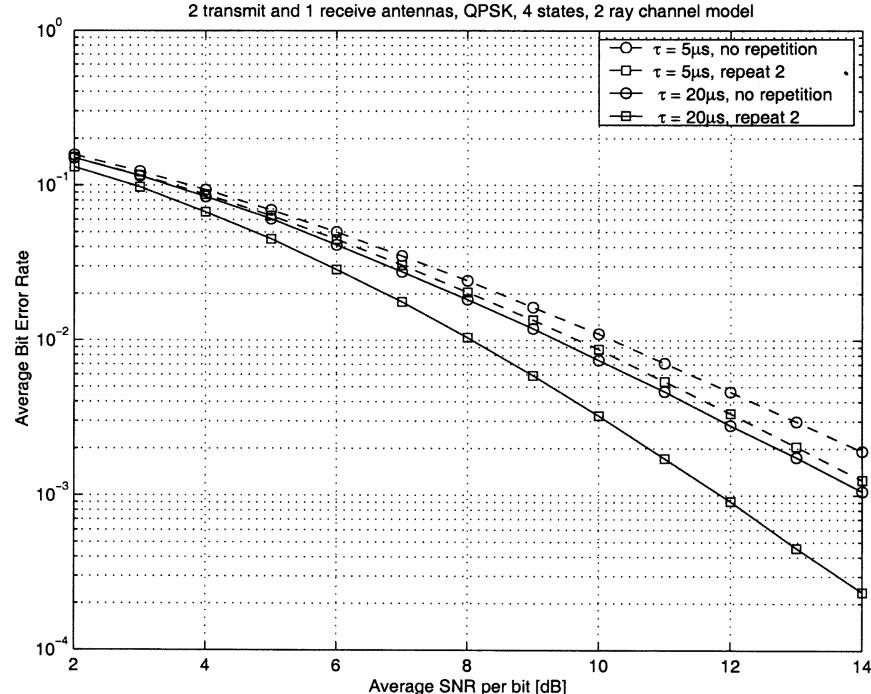

Fig. 5. Performance of two-antenna SF trellis codes with the two-ray channel model.

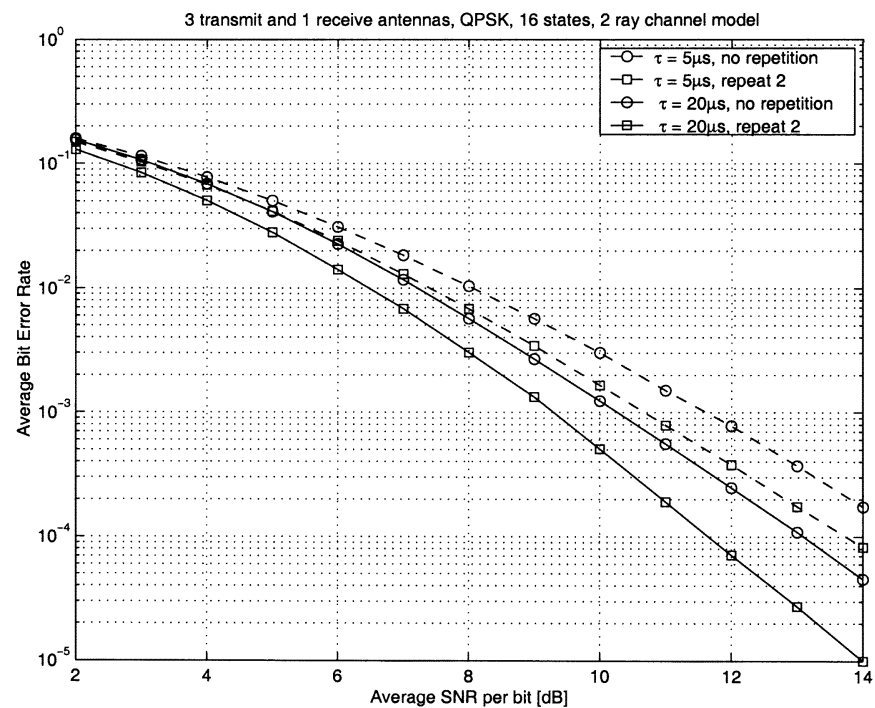

Fig. 6. Performance of three-antenna SF trellis codes with the two-ray channel model.

was $((128 \times 3 / 4 \mathrm{~b}) /((128 \mu \mathrm{s}+20 \mu \mathrm{s}) \times 1 \mathrm{MHz}))=0.65 \mathrm{~b} / \mathrm{s} / \mathrm{Hz}$. The tendencies observed in Fig. 4 are similar to those observed in Fig. 3. In case of $\tau=20 \mu$ s, the full-diversity SF code has a steeper performance curve than the SF code using ST without repetition, and it has an improvement of about $1 \mathrm{~dB}$ at a BER of $10^{-4}$. In the case of $\tau=5 \mu \mathrm{s}$, the performance of the proposed SF code is a little worse than that of the SF code using ST code without repetition. The worse performance is due to the smaller coding advantage of the proposed SF code since, in order to keep the same spectral efficiency of the two schemes, we used QPSK modulation for the proposed SF code and BPSK modulation for the nonrepeated code.

The performance of the SF trellis codes for two and three transmit antennas are depicted in Figs. 5 and 6, respectively. We can see that the full-diversity SF trellis codes have better diversity order than the nonrepeated codes in case of the longer delay profile (i.e., $\tau=20 \mu \mathrm{s}$ ). At a BER of $10^{-3}$, there is an improvement of about $2 \mathrm{~dB}$ for the two-antenna case and an improvement of 
about $1 \mathrm{~dB}$ for the three-antenna case. The modulation scheme was QPSK for all trellis codes; therefore, the nonrepeated codes achieved a spectral efficiency of $1.73 \mathrm{~b} / \mathrm{s} / \mathrm{Hz}$, whereas the repeated codes had a spectral efficiency of $0.86 \mathrm{~b} / \mathrm{s} / \mathrm{Hz}$. Note that Figs. 5 and 6 demonstrate that the proposed SF code construction method can be used to produce a set of codes that have different rates and error correction capabilities, offering different tradeoffs between code rate and diversity order.

Based on the above simulation results, we can make some observations. It is apparent that by repeating each row of the space-time code matrix, we could construct codes whose error performance curve is steeper than that of the codes without repetition, i.e., the obtained codes have higher diversity order. However, the actual performance of the code depends heavily on the underlying channel model. In all cases, both the absolute performance and the performance improvement obtained by repetition are considerably better in case of the longer delay profile (i.e., $\tau=20 \mu \mathrm{s}$ ), and the performance of the obtained full-diversity $\mathrm{SF}$ codes degrade significantly in case of the delay profile with $\tau=5 \mu \mathrm{s}$. These phenomena can be explained as follows.

The delay distribution of the channel has a significant effect on the SF code performance. If the delays of the paths are large with respect to one OFDM block period, there will be fast variations in the spectrum of the channel impulse response; therefore, the probability of simultaneous deep fades in adjacent subchannels will be smaller. This observation is in accordance with Theorem 2. As discussed in Section V, we should expect better BER performance when transmitting data over channels with larger path delays. On the other hand, if the two delay paths are very close, the channel will cause performance degradation.

\section{B. Simulations With the COST207 Six-Ray Delay Profile}

The second set of experiments were performed using a more realistic channel model. The SF codes simulated in the previous subsection were tested over the COST207 Typical Urban (TU) six-ray channel model [34]. The power delay profile of the channel is shown in Fig. 7. We simulated an MIMO-OFDM system having $N=128$ subcarriers with two different bandwidths: i) $B W=1 \mathrm{MHz}$ (denoted by dashed lines), and ii) $B W=4 \mathrm{MHz}$ (denoted by solid lines). The cyclic prefix was $20 \mu \mathrm{s}$ long for both cases. In all of the simulations, the curves with squares (" $\square$ ") show the performance of the SF codes obtained by repeating each row of the corresponding space-time codes two times, and the curves with circles ("o") show the results for the case of using ST codes as SF codes (without repetition).

The performance of the SF block codes from the $2 \times 2$ orthogonal design with and without repetition are shown in Fig. 8 for two transmit and one receive antennas. The repeated code (using QPSK modulation) and the nonrepeated code (using BPSK modulation) had the same spectral efficiency of $0.86 \mathrm{~b} / \mathrm{s} / \mathrm{Hz}$ for the $1-\mathrm{MHz}$ system and $0.22 \mathrm{~b} / \mathrm{s} / \mathrm{Hz}$ for the 4-MHz system, respectively. We can see from the figure that in case of $B W=4 \mathrm{MHz}$, the code with repetition has a steeper performance curve than the code without repetition. There is a performance improvement of about $2 \mathrm{~dB}$ at a BER of $10^{-4}$. In case of $B W=1 \mathrm{MHz}$, the maximum delay of the TU profile $(5.0 \mu \mathrm{s})$ is "short" compared to the "long" duration of

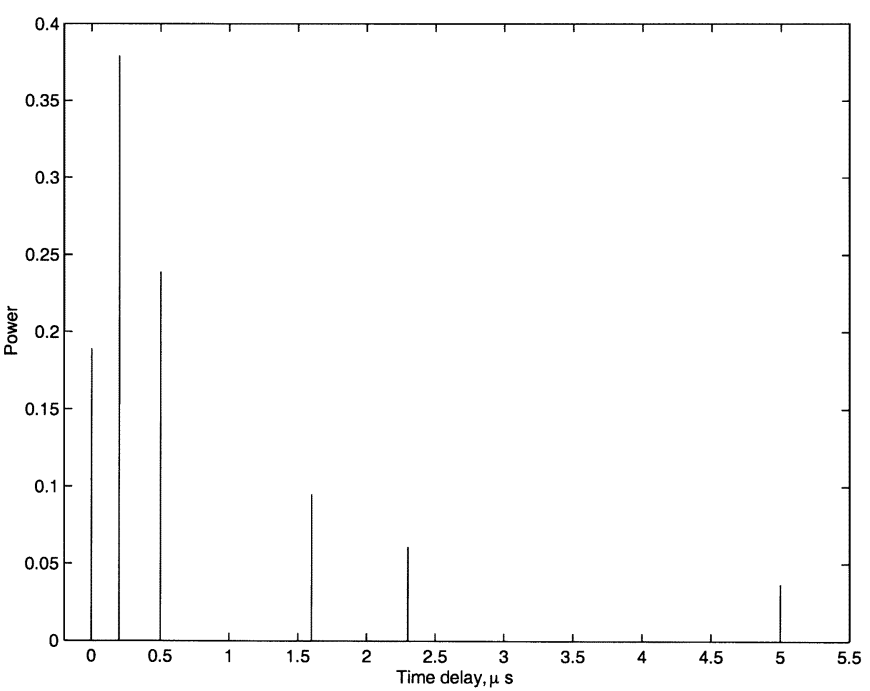

Fig. 7. COST207 Typical Urban (TU) six-ray power delay profile.

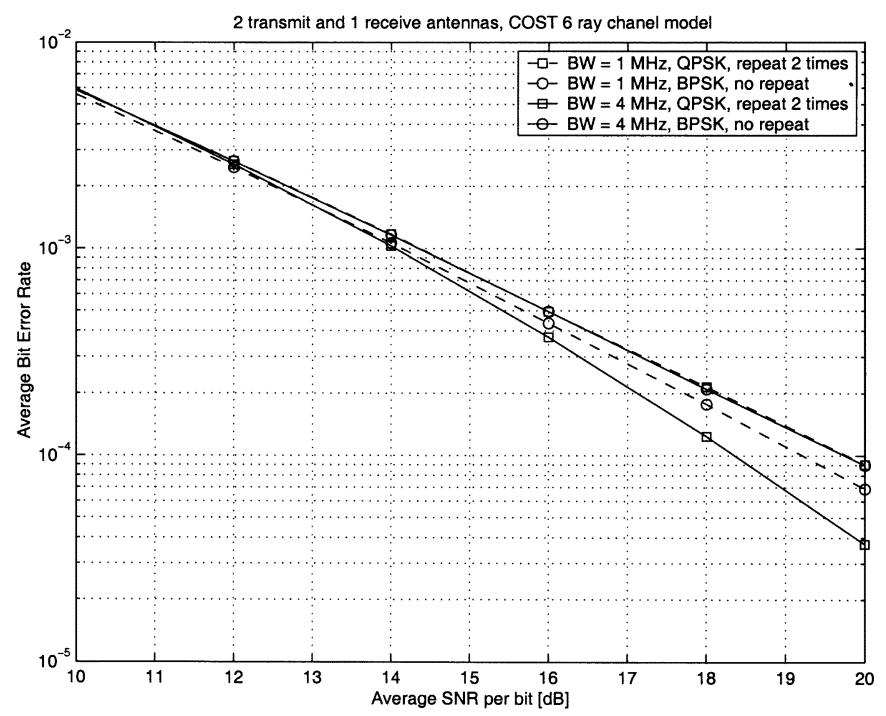

Fig. 8. Performance of two-antenna SF block codes with the COST six-ray channel model.

the OFDM block $(128 \mu \mathrm{s})$, which means that there is little frequency diversity available in the fading channel. From the figure, we observe that the performance of the repeated code is worse than the nonrepeated code, due to the smaller coding advantage, which is a result of the larger constellation size.

The rest of the figures show the BER curves of different SF trellis codes. In case of the 1-MHz MIMO-OFDM system, the spectral efficiencies of the trellis codes were $1.73 \mathrm{~b} / \mathrm{s} / \mathrm{Hz}$ (no repetition) and $0.86 \mathrm{~b} / \mathrm{s} / \mathrm{Hz}$ (repeat twice). The $4-\mathrm{MHz}$ system yielded the spectral efficiencies $0.43 \mathrm{~b} / \mathrm{s} / \mathrm{Hz}$ (no repetition) and $0.22 \mathrm{~b} / \mathrm{s} / \mathrm{Hz}$ (repeat twice). The performance of the QPSK SF trellis code with and without repetition can be observed in Fig. 9 for two transmit and one receive antennas, in Fig. 10 for two transmit and two receive antennas, and in Fig. 11 for three transmit and one receive antennas. We can see that in case of $B W=4 \mathrm{MHz}$, all of the $\mathrm{SF}$ trellis codes with repetition have higher diversity order than those without repetition. However, in case of $B W=1 \mathrm{MHz}$, there is almost no diversity advantage over the nonrepeated case. 


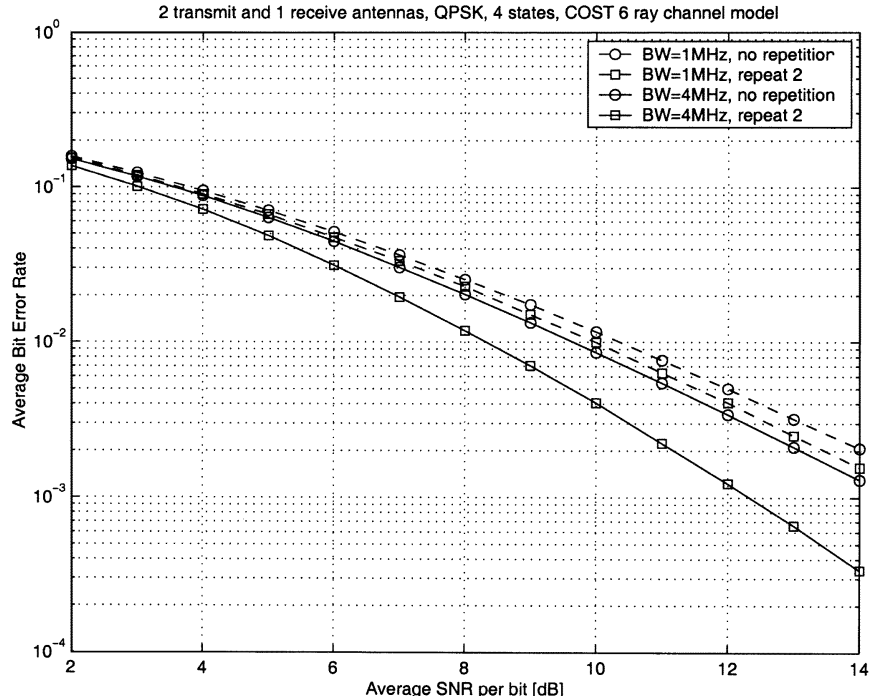

Fig. 9. Performance of two-antenna SF trellis codes with the COST six-ray channel model.

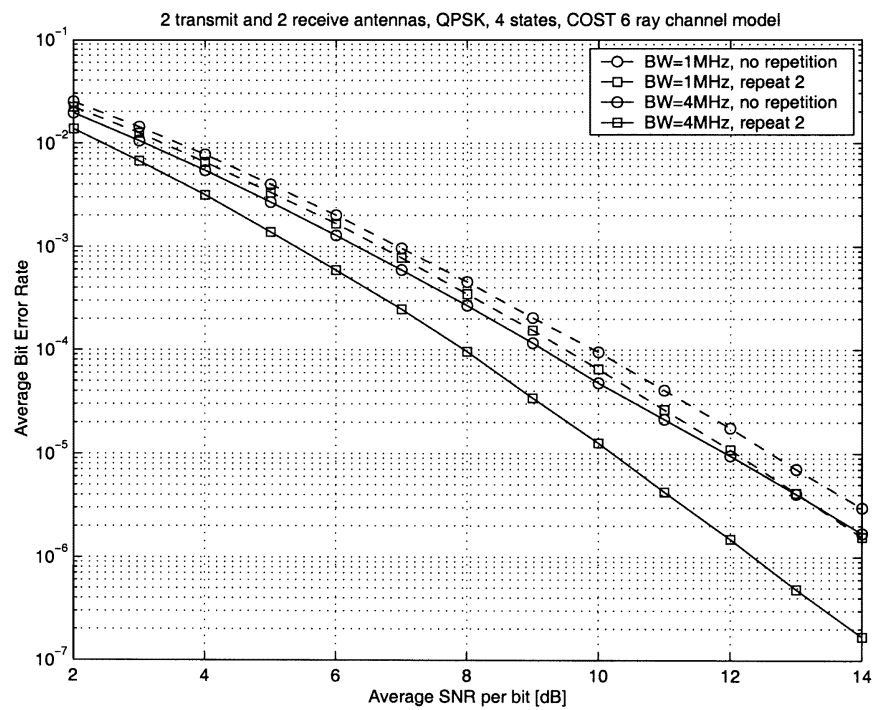

Fig. 10. Performance of two-antenna SF trellis codes with two receive antennas and the COST six-ray channel model.

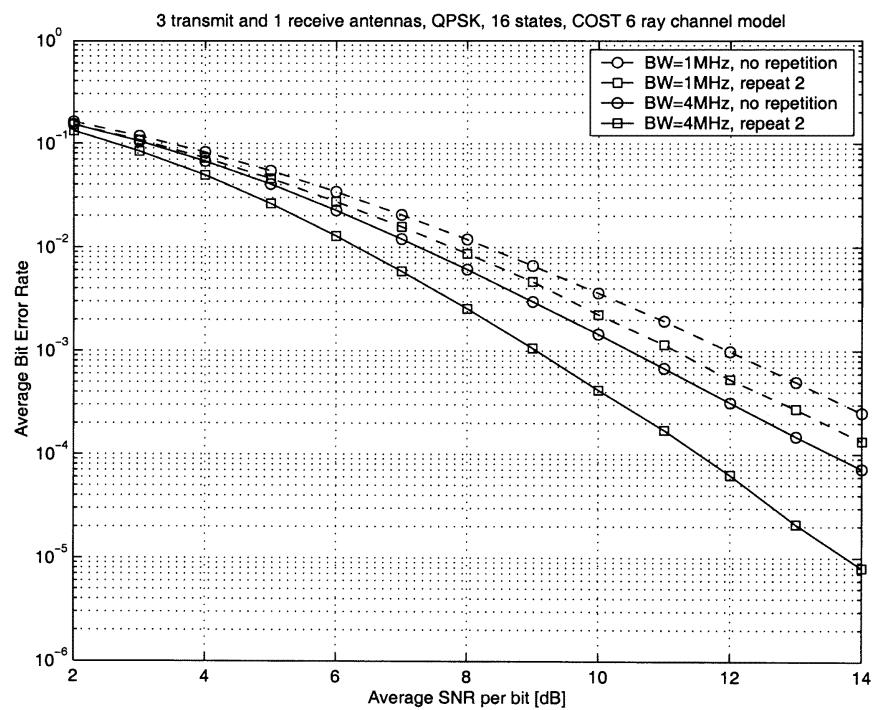

Fig. 11. Performance of three-antenna SF trellis codes with the COST six-ray channel model.
The tendencies of the performance curves obtained with the more realistic TU profile are similar to those observed in the first set of experiments with the two-ray profiles. As the bandwidth increases, the delays become longer relative to the OFDM block period, resulting in more significant performance improvement. If the ratio of the maximum path delay and the OFDM block duration is small, the behavior of the channel will be similar to that of a flat fading channel.

\section{CONCLUSION}

We proposed a systematic SF code design method for MIMO-OFDM systems with arbitrary power delay profiles. The full-diversity SF codes were constructed from ST codes via a simple repetition mapping. This mapping is independent of the particular properties of the applied ST codes; therefore, all the existing ST block and trellis codes achieving full spatial diversity in quasistatic flat fading environment can be used to achieve the maximum diversity available in frequency-selective MIMO fading environment. We also characterized the performance of the SF codes obtained via the mapping by finding the lower and upper bounds on their coding advantages as functions of the coding advantages of the underlying ST codes.

Based on the theoretical and simulation results, we can draw the following conclusions. First, the performance of the SF codes designed by our method depends on the performance of the underlying ST code. Second, the actual performance of the SF codes also depends on the delay distribution and the power distribution of the channel impulse responses. Third, the repetition mapping in the code construction method causes a coding rate decrease compared with the underlying ST codes. However, to our knowledge, the proposed SF codes have the best known coding rate of all SF codes that are guaranteed to achieve full diversity.

\section{REFERENCES}

[1] J.-C. Guey, M. P. Fitz, M. R. Bell, and W.-Y. Kuo, "Signal design for transmitter diversity wireless communication systems over Rayleigh fading channels," IEEE Trans. Commun., vol. 47, pp. 527-537, Apr. 1999.

[2] V. Tarokh, N. Seshadri, and A. R. Calderbank, "Space-time codes for high data rate wireless communication: performance criterion and code construction," IEEE Trans. Inform. Theory, vol. 44, pp. 744-765, Apr. 1998.

[3] S. Alamouti, "A simple transmit diversity technique for wireless communications," IEEE J. Select. Areas Commun., vol. 16, pp. 1451-1458, Aug. 1998.

[4] V. Tarokh, H. Jafarkhani, and A. R. Calderbank, "Space-time block codes from orthogonal designs," IEEE Trans. Inform. Theory, vol. 45, pp. 1456-1467, Oct. 1999.

[5] B. M. Hochwald and T. L. Marzetta, "Unitary space-time modulation for multiple-antenna communication in Rayleigh flat fading," IEEE Trans. Inform. Theory, vol. 46, pp. 543-564, Feb. 2000

[6] B. M. Hochwald, T. L. Marzetta, T. J. Richardson, W. Swelden, and R. Urbanke, "Systematic design of unitary space-time constellations," IEEE Trans. Inform. Theory, vol. 46, pp. 1962-1973, June 2000.

[7] B. L. Hughes, "Differential space-time modulation," IEEE Trans. Inform. Theory, vol. 46, pp. 2567-2578, Nov. 2000.

[8] B. M. Hochwald and W. Sweldens, "Differential unitary space-time modulation," IEEE Trans. Commun., vol. 48, pp. 2041-2052, Dec. 2000.

[9] A. Shokrollahi, B. Hassibi, B. M. Hochwald, and W. Sweldens, "Representation theory for high-rate multiple-antenna code design," IEEE Trans. Inform. Theory, vol. 47, pp. 2335-2367, Sept. 2001.

[10] W. Su and X. G. Xia, "On space-time block codes from complex orthogonal designs," Wireless Pers. Commun., vol. 25, pp. 1-26, Apr. 2003.

[11] Q. Yan and R. Blum, "Optimum space-time convolutional codes," in Proc. IEEE WCNC, vol. 3, 2000, pp. 1351-1355.

[12] _ _ "Robust space-time block coding for rapid fading channels," in Proc. IEEE GLOBECOM, vol. 1, 2001, pp. 460-464. 
[13] Z. Safar and K. J. R. Liu, "Systematic design of space-time trellis codes for diversity and coding advantages," EURASIP J. Applied Signal Processing, no. 3, pp. 221-235, Mar. 2002.

[14] L. J. Cimini, "Analysis and simulation of a digital mobile channel using orthogonal frequency division multiplexing," IEEE Trans. Commun., vol. COMM-33, pp. 665-675, July 1985 .

[15] Y. Li, L. J. Cimini, and N. R. Sollenberger, "Robust channel estimation for OFDM systems with rapid dispersive fading channels," IEEE Trans. Commun., vol. 46, pp. 902-915, July 1998.

[16] Y. Li, J. H. Winters, and N. R. Sollenberger, "MIMO-OFDM for wireless communications: signal detection with enhanced channel estimation," IEEE Trans. Commun., vol. 50, pp. 1471-1477, Sept. 2002.

[17] D. Agrawal, V. Tarokh, A. Naguib, and N. Seshadri, "Space-time coded OFDM for high data-rate wireless communication over wideband channels," in Proc. IEEE VTC, vol. 3, 1998, pp. 2232-2236.

[18] K. Lee and D. Williams, "A Space-frequency transmitter diversity technique for OFDM systems," in Proc. IEEE GLOBECOM, vol. 3, 2000, pp. 1473-1477.

[19] R. Blum, Y. Li, J. Winters, and Q. Yan, "Improved space-time coding for MIMO-OFDM wireless communications," IEEE Trans. Commun., vol. 49, pp. 1873-1878, Nov. 2001.

[20] Y. Gong and K. B. Letaief, "An efficient space-frequency coded wideband OFDM system for wireless communications," in Proc. IEEE ICC, vol. 1, 2002, pp. 475-479.

[21] Z. Hong and B. Hughes, "Robust space-time codes for broadband OFDM systems," in Proc. IEEE WCNC, vol. 1, 2002, pp. 105-108.

[22] H. Bölcskei and A. J. Paulraj, "Space-frequency coded broadband OFDM systems," in Proc. IEEE WCNC, Sept. 2000, pp. 1-6.

[23] - "Space-frequency codes for broadband fading channels," in Proc. ISIT, Washington, DC, June 24-29, 2001, p. 219.

[24] B. Lu and X. Wang, "Space-time code design in OFDM systems," in Proc. IEEE GLOBECOM, Nov. 2000, pp. 1000-1004.

[25] Y. Gong and K. B. Letaief, "Space-frequency-time coded OFDM for broadband wireless communications," in Proc. IEEE GLOBECOM, San Antonio, TX, Nov. 2001.

[26] Z. Liu, Y. Xin, and G. Giannakis, "Space-time-frequency coded OFDM over frequency selective fading channels," IEEE Trans. Signal Processing, vol. 50, pp. 2465-2476, Oct. 2002.

[27] S. Zhou and G. Giannakis, "Space-time coding with maximum diversity gains over frequency selective fading channels," IEEE Signal Processing Lett., vol. 8, pp. 269-272, Oct. 2001.

[28] Y. Liu, M. P. Fitz, and O. Y. Takeshita, "Space-time codes performance criteria and design for frequency selective fading channels," in Proc. IEEE ICC, vol. 9, Helsinki, Finland, June 11-15, 2001, pp. 2800-2804.

[29] M. P. Fitz, J. Grimm, and S. Siwamogsatham, "A new view of performance analysis techniques in correlated Rayleigh fading," in Proc. IEEE WCNC, Sept. 1999, pp. 139-144.

[30] S. Siwamogsatham, M. P. Fitz, and J. Grimm, "A new view of performance analysis of transmit diversity schemes in correlated Rayleigh fading," IEEE Trans. Inform. Theory, vol. 48, pp. 950-956, Apr. 2002.

[31] W. Su, Z. Safar, and K. J. R. Liu, "Space-time signal design for timecorrelated Rayleigh fading channels," in Proc. IEEE ICC, vol. 5, May 2003, pp. 3175-3179.

[32] R. A. Horn and C. R. Johnson, Matrix Analysis. Cambridge, U.K.: Cambridge Univ. Press, 1985.

[33] —-, Topics in Matrix Analysis. Cambridge, U.K.: Cambridge Univ. Press, 1991

[34] G. Stuber, Principles of Mobile Communication. Boston, MA: Kluwer, 2001.

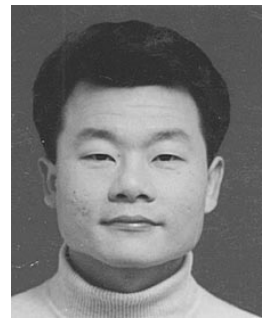

Weifeng Su (M'03) received the B.S. and Ph.D. degrees in mathematics from Nankai University, Tianjin, China, in 1994 and 1999, respectively, and the $\mathrm{Ph} . \mathrm{D}$. degree in electrical engineering from the University of Delaware, Newark, in 2002.

From 1994 to 1999, he was a Research Assistant with Nankai University. From 1999 to 2002, he was a Research Assistant with the Department of Electrical and Computer Engineering, University of Delaware. Currently, he is a Postdoctoral Research Associate with the Institute for Systems Research, University of Maryland, College Park. His current research interests include wireless communication systems and information theory, especially in space-time coding and space-frequency coding for MIMO-OFDM systems.

Dr. Su received the Competitive Fellowship Award and the Signal Processing and Communications Faculty Award from the University of Delaware in 2001 and 2002, respectively.

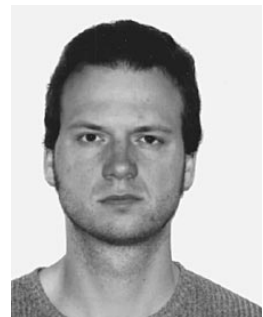

Zoltan Safar received the University Diploma in electrical engineering from the Technical University of Budapest, Budapest, Hungary, in 1996 and the M.S. and Ph.D. degrees in electrical engineering from the University of Maryland, College Park, in 2001 and 2003, respectively.

Currently, he is an assistant professor with the Department of Innovation, IT University of Copenhagen, Copenhagen, Denmark. His research interests include wireless communications and multimedia signal processing, with particular focus on MIMO systems, space-time and space-frequency coding.

Dr. Safar was the recipient of the Outstanding Systems Engineering Graduate Student Award from the Institute for Systems Research, University of Maryland, in 2003.

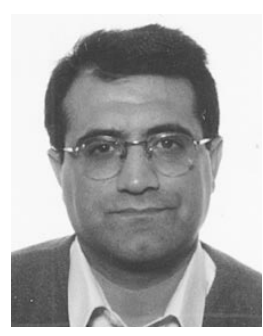

Masoud Olfat ( $\mathrm{S}^{\prime} 95$ ) received the B.S. degree in electrical engineering from Sharif University of Technology, Tehran, Iran, in 1993 and the M.S. degree in electrical engineering from the University of Maryland, College Park, in 1998. He is currently pursuing the Ph.D. degree in electrical engineering at the University of Maryland, College Park.

From October 1995 to August 1996, he worked at CASE center, Syracuse, NY. In 1998, he joined the Odyssey Technology Inc., Laurel, MD, as a member of technical staff. He joined the Hermes Technologies Inc., Potomac, MD, in 2001, working with the IEEE802.11a development team. His current research interests include array and statistical signal processing, coding, power control, and space-time and space-frequency coding for wireless communications, with a special interest in OFDM systems.

Mr. Olfat ranked first in the Iranian universities nationwide entrance examination in the western provinces of Iran in 1984.

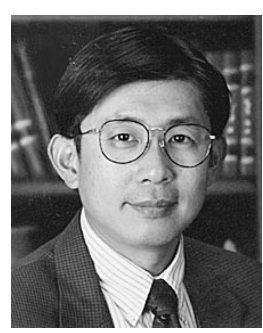

K. J. Ray Liu (F'03) received the B.S. degree from the National Taiwan University, Taipei, Taiwan, R.O.C., in 1983 and the Ph.D. degree from the University of California, Los Angeles, in 1990, both in electrical engineering.

$\mathrm{He}$ is a Professor with the Electrical and Computer Engineering Department and Institute for Systems Research, University of Maryland, College Park. His research interests span broad aspects of signal processing algorithms and architectures, multimedia communications and signal processing, wireless communications and networking, information security, and bioinformatics.

Dr. Liu is the recipient of numerous honors and awards, including the IEEE Signal Processing Society 2004 Distinguished Lecturer, the 1994 National Science Foundation Young Investigator Award, the IEEE Signal Processing Society's 1993 Senior Award (Best Paper Award), and the IEEE 50th Vehicular Technology Conference Best Paper Award (Amsterdam, the Netherlands, 1999). He also received the George Corcoran Award in 1994 for outstanding contributions to electrical engineering education and the Outstanding Systems Engineering Faculty Award in 1996 in recognition of outstanding contributions in interdisciplinary research, both from the University of Maryland. He is the Editor-in-Chief of IEEE SIGNAL PROCESSING MAGAZINE and was the Editor-inChief of EURASIP Journal on Applied Signal Processing. He has served as an Associate Editor of IEEE TRANSACTIONS ON Signal Processing, a Guest Editor of special issues on Multimedia Signal Processing of PROCEEDINGS OF THE IEEE, a Guest Editor of a special issue on Signal Processing for Wireless Communications of the IEEE JOURNAL OF SELECTED AREAS IN COMMUNICATIONS, a Guest Editor of the special issue on Multimedia Communications over Networks of the IEEE Signal PRocessing MAGAZINE, a Guest Editor of special issue on Multimedia over IP of IEEE TRANSACTIONS ON MULTIMEDIA, and an editor of the Journal of VLSI Signal Processing Systems. He has served as Chairman of the Multimedia Signal Processing Technical Committee of the IEEE Signal Processing Society. 